\title{
Essais de rationnement de boucs de la race rousse de Maradi avec des sous-produits de l'agriculture locale
}

\author{
par G. PLON $\left(^{*}\right)$
}

\begin{abstract}
RÉSUMÉ
L'auteur montre qu'il est possible d'assurer l'entretien ou la croissance de boucs de cette race, en fin de saison sèche alors que les pâturages naturels ont pratiquement disparu, en les alimentant exclusivement avec des sous-produits de l'agriculture locale : graines de coton ; fanes sèches de Niebé ; son de mil ; tourteau d'arachide, avec des rations dont 1 donne la composition et le prix de revient.
\end{abstract}

\section{GÉNÉRALITÉS}

La chèvre rousse de Maradi occupe une place importante dans l'élevage au Niger du fait de sa prolificité et des qualités remarquables de sa peau, dont le grain est très apprécié dans la peausserie de luxe.

C'est pourquoi les services de l'Elevage et de la Reconstitution du cheptel s'efforcent d'en augmenter l'aire de diffusion partout où les conditions climatiques, pastorales et écologiques paraissent favorables.

Mais cette diffusion est freinée par certaines caractéristiques de cette chèvre :

- elle est beaucoup moins résistante que la chèvre sahélienne aux rigueurs de l'environnement, notamment en fin de saison sèche ;

- sédentaire, elle doit trouver sa pâture à proximité du village ce qui implique, lorsque les fourrages naturels se font rares, des soins particuliers de la part de son propriétaire, surtout en ce qui concerne son alimentation.

En fait, cette chèvre ne prospère vraiment bien qu'en zone soudano-sahélienne - là où actuellement les cultures vivrières et industrielles se

(") Bureau du projet ITURI, Ambassade de France MC/BDPA, B. P. 7861, Kinshasa/Gombê, Zaïre. développent régulièrement avec les réductions de pâtures naturelles qui en résultent.

Dans ces conditions, l'utilisation des sousproduits agricoles locaux est devenue essentielle pour assurer son alimentation à la période de soudure, notamment lorsque la saison sèche se termine.

C'est pourquoi 3 essais de rationnement ont été menés avec ces sous-produits: graine de coton; fanes sèches de Niébé (Vigna sinensis); son de mil et tourteau d'arachide, dans des conditions similaires, au Centre caprin de Maradi, sans lots d'animaux témoins.

Cette absence tient à 2 faits :

- ce Centre, qui a pour mission essentielle de produire des boucs sélectionnés, n'est pas équipé pour faire de la recherche ;

- son troupeau bénéficie de conditions d'alimentation (pâture et supplémentation) nettement supérieures à celles que connaissent les caprins des éleveurs locaux.

En conséquence, ces essais sont seulement indicatifs avec estimation chiffrée des possibilités de rationnement avec ces sous-produits à l'époque où les disponibilités fourragères naturelles deviennent de plus en plus rares, avec le prix de revient des rations proposées. 
Plantes appétées pur les chèvres du Centre Caprin de Maŕdı.

\begin{tabular}{|c|c|c|c|}
\hline Nom de la plante & Parties appétēes & Appétence & $\begin{array}{c}\text { Frëquence } \\
\text { (sur la station) }\end{array}$ \\
\hline Acacia ataxantha & Feuilles et graines & ++ & G \\
\hline Albizzia chevalieri & Feuilles et fruits & ++ & F \\
\hline Andropogon gayanus & Feuilles & ++ & $M$ \\
\hline Aristida stipoides & Feuilles & ++ & $F$ \\
\hline Blepharis linarii fotia & Graines, ur peu les feuilles & ++ & G \\
\hline Boseia senegatensis & Feuilles & 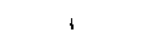 & $\mathrm{T}$ \\
\hline Cassia bingueana & Feuilles & ++ & M \\
\hline Ceratotheoa sesamoides & Feuilles et graines & ++ & $\mathrm{T}$ \\
\hline Comiphora africana & Feuilles et fruits & +++ & T \\
\hline Cochtospernum planchonit & Feuilles puis tiges & ++ & G \\
\hline Dactyloctenium aegyptium & Jeunes pousses et pailles & ++ & F \\
\hline Detarium microcarpum & Feuilles & ++ & $\mathrm{T}$ \\
\hline Exagrostis tremula & Petites feuilles et ēpillets & ++ & G \\
\hline Guiera senegalensis & Feuilles vertes, fleurs & ++ & G \\
\hline Hibiscus sabdariffo & Toute la plante & ++ & $\mathrm{T}$ \\
\hline Indigofera Sp. & Feuilles & ++ & $\mathrm{F}$ \\
\hline Kigetia africana & Feuilles & + & $\mathrm{M}$ \\
\hline Konautia Spp. & Feuilles et graines & ++ & $\mathrm{F}$ \\
\hline Lipidagatis arrobryia & Vert et pailie, toute 1 a plante & ++ & F \\
\hline Maema crassifoitia & Feuilles, fleurs, fruits & ++ & $\mathrm{T}$ \\
\hline Mermenia pinnaca & Feuilles (S.P.), fruits (S.S.) & ++ & M \\
\hline Witracarpus villosus & Toute la plante & ++ & $\mathrm{F}$ \\
\hline Piliostigma rètiuulatum & Feuilles, fruits & ++ & $\mathrm{M}$ \\
\hline Polygala erioptera & Vert et paille, toute la plante & ++ & $G$ \\
\hline Prosopis africana & Fruits, un peu les feuilles & ++ & $\mathrm{M}$ \\
\hline Pterocarpus erinacens & Feuilles & ++ & F \\
\hline Pupalia lapacea & Feuilles, fleurs & ++ & $E$ \\
\hline Scïerocarya birrea & Eeuilles, fruits & +++ & M \\
\hline Stytosanthes mucronata & Feuilles, fleurs & ++ & M \\
\hline Tephrosia bracteolata & Feuilles, fruits & ++ & F \\
\hline Tephrosia Zinearis & Feuilles, gousses & ++ & M \\
\hline Terminalia avicennioides & Feuilles & ++ & $\mathrm{F}$ \\
\hline Ximenic americana & Feuilles, fruits & ++ & M \\
\hline
\end{tabular}

$+=$ un peu ; ++ = bien ; +++ = trẽs bien. $\quad T=$ Traces ; $F=$ Faible ; $M=$ Moyenne ; $G=$ Grande. 


\section{Rationnement avec de la graine de coton}

a) Objet de l'essai

Il a eu pour objet de préciser la valeur nutritive de ces graines et de vérifier leur absence de toxicité.

En 1976, en effet, après une saison des pluies très déficitaire, 10 boucs castrés ont dû être entretenus pendant 4 mois de saison sèche (de janvier à mai) sur 4 ha de pâturage sec et rare en n'ayant pratiquement à consommer que des graines de coton, distribuées à volonté.

A la fin de cette période, au cours de laquelle aucun trouble digestif n'a été observé, les animaux étaient en bon état d'entretien et avaient gardé leur poids initial.

\section{b) Conditions de l'essai}

Dix mâles d'un an environ, porteurs de leurs dents de lait, en bon état, d'un poids moyen de $19,260 \mathrm{~kg}$, sélectionnés parmi les animaux du Centre ont été isolés pendant 1 mois, dans un parc de $600 \mathrm{~m}^{2}$ dépourvu de toute végétation, avec comme seule nourriture des graines de coton entières, non délintées et de l'eau à volonté.

Ils ont fait l'objet d'une triple pesée individuelle en début et en fin d'essai avec le poids moyen retenu, aux 15 février et 15 mars 1977 et de pesées hebdomadaires simples de contrôle.

\section{c) Résultats}

Ils ont été les suivants :

\begin{tabular}{|c|c|c|c|}
\hline & \multicolumn{3}{|c|}{ Poids en kg } \\
\hline $\mathrm{N}^{\circ}$ & au 15.2 .1977 & au 15.3 .1977 & $\begin{array}{c}\text { Variation } \\
\text { pondérale } \\
\text { en kg }\end{array}$ \\
\hline 1 & 17,2 & 17,00 & $-0,2$ \\
2 & 20,00 & 19,6 & $-0,4$ \\
3 & 22,6 & 22,00 & $-0,6$ \\
4 & 20,8 & 21,00 & $+0,2$ \\
5 & 17,2 & 17,6 & $+0,4$ \\
6 & 23,6 & 24,00 & $+0,4$ \\
7 & 16,00 & 17,00 & $+1,0$ \\
8 & 14,5 & 16,00 & $+1,5$ \\
9 & 22,5 & 24,00 & $+1,5$ \\
10 & 18,2 & 20,00 & $+1,8$ \\
\hline Poids & 192,6 & 199,2 & $+5,6$ \\
total & & & + \\
\hline
\end{tabular}

Au cours de l'essai, $150 \mathrm{~kg}$ de graines de coton ont été consommés, soit une consommation moyenne de $0,536 \mathrm{~kg}$ de graine par animal et par jour. d) Commentaires

Ces $0,536 \mathrm{~kg}$ ont apporté, à chaque animal (1)
U. F.
0,516
M. S
$496,34 \mathrm{~g}$
M. A. D.
$82,4 \quad \mathrm{~g}$
$\mathrm{Ca}$
$0,9 \quad \mathrm{~g}$
$\mathbf{P} \ldots \ldots \ldots \ldots \ldots$
$1,78 \mathrm{~g}$

Pour un caprin de $20 \mathrm{~kg}$, il est admis (1) :

- que la consommation volontaire traduite en matière sèche est de $0,5 \mathrm{~kg} / \mathrm{j}$;

- que ses besoins d'entretien sont de :
U. F. . . . . . . . . 0,50
M. A. D. ........ $35 \mathrm{~g}$
$\mathrm{Ca} \ldots \ldots \ldots \ldots \ldots \ldots \ldots \ldots, \quad 1,00 \mathrm{~g}$
P............ $0,60 \mathrm{~g}$

Les besoins d'entretien de ces 10 boucs ont donc été entièrement couverts, ce qui est confirmé par des résultats pondéraux qui montrent que ces animaux ont conservé leur poids en 4 semaines, avec même pour certains un léger accroissement de poids non significatif.

Il convient de remarquer que cette ration, si elle a assuré l'entretien de tous ces mâles, n'a permis la croissance que de 4 d'entre eux seulement, avec un gain moyen de poids de $1,300 \mathrm{~kg}$.

Peut-être peut-on en tirer la conclusion que ces derniers se sont mieux ou plus vite adaptés que les autres à la graine de coton, ou qu'ils en ont consommé davantage ou qu'ils l'ont mieux utilisée.

Dans ce domaine, de nombreuses hypothèses sont possibles et seules de véritables recherches pourraient permettre d'en circonscrire les raisons.

Aucun trouble digestif n'est apparu, ce qui confirme l'intérêt de la graine de coton en cas de pénurie de fanes de Niébé, intérêt d'autant plus grand qu'il s'agit là d'un aliment de rechange de qualité et d'utilisation économique puisque cette graine valait à l'époque $11 \mathrm{~F} \mathrm{CFA} \mathrm{le} \mathrm{kg}$.

Les éleveurs en sont d'ailleurs si convaincus que depuis, la demande en graine excède en permanence l'offre, avec pénurie constante de ce sous-produit.

2. Rationnement avec des fanes sèches de Niébé, du foin et du tourteau d'arachide.

\section{a) Objet de l'essai}

Il a eu pour objet d'établir la valeur alimentaire en saison sèche d'une ration d'entretien à 
base de fanes de Niébé et de foin enrichie par du tourteau d'arachide.

\section{b) Conditions de l'essai}

Au Centre caprin de Maradi, le troupeau dispose d'un pâturage herbacé et arbustif, dont la composition est donnée en annexe, qui suffit pour assurer ses besoins alimentaires, sauf en fin de saison sèche. A cette époque, une supplémentation de fanes de Niébé est distribuée au troupeau sur la base de $800 \mathrm{~g}$ par animal et par jour jusqu'à la repousse du pâturage naturel.

L'essai a donc consisté à fournir aux animaux une ration d'entretien équivalant à peu de chose près à celle dont dispose à la même époque le troupeau du Centre avec du tourteau d'arachide en supplément pour étudier son éventuel effet bénéfique sur des boucs n'ayant pas encore atteint leur poids maximal.

Les 10 boues adultes ( 2 dents) en bon état, d'un poids moyen de $26,250 \mathrm{~kg}$, choisis pour cet essai, ont été isolés pendant 2 mois (du 14 mai au 14 juillet) dans un parc clôturé de $600 \mathrm{~m}^{2}$, totalement dépourvu de végétation, avec de l'eau à volonté et une ration globale quotidienne ainsi composée :

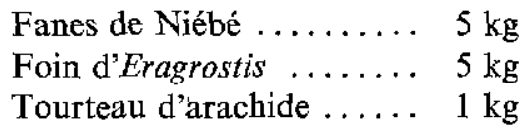

Chaque bouc a fait l'objet d'une triple pesée en début et en fin d'essai, ainsi qu'une pesée hebdomadaire simple. Aucune maladie ni aucune mortalité ne sont venues troubler son déroulement.

\section{c) Résultats}

Ces résultats sont donnés dans le tableau suivant :

\begin{tabular}{|r|c|c|c|}
\hline & \multicolumn{2}{|c|}{ Poids en $\mathrm{kg}$} & $\begin{array}{c}\text { Résultat } \\
\text { Einal } \\
\text { en } \mathrm{kg}\end{array}$ \\
\cline { 2 - 4 } & $\begin{array}{c}\text { en début de } \\
\text { 1'essai (14.5) }\end{array}$ & $\begin{array}{c}\text { en fin } \mathrm{fin} \\
\mathrm{d} \text { essai (145) }\end{array}$ & - \\
\hline 1 & 27,50 & 25,80 & $-1,7$ \\
2 & 26,90 & 25,30 & $-1,6$ \\
3 & 28,40 & 28,00 & $-0,4$ \\
4 & 20,80 & 20,70 & $-0,1$ \\
5 & 31,20 & 32,20 & $+1,0$ \\
6 & 23,80 & 25,00 & $+1,20$ \\
7 & 26,1 & 27,50 & $+1,40$ \\
8 & 24,80 & 27,30 & $+2,50$ \\
9 & 23,50 & 26,70 & $+3,20$ \\
10 & 29,50 & 35,00 & $+5,50$ \\
\hline Tota1 & 262,50 & 273,50 & $+11,00$ \\
\hline
\end{tabular}

En 2 mois, le gain total a été de $11 \mathrm{~kg}$ soit $1,100 \mathrm{~kg}$ en moyenne par animal, ce qui paraît satisfaisant.

Au niveau du troupeau :

- deux boucs ont maigri ;

- deux ont conservé leur poids, ou à peu près ;

- six ont grossi ;

avec le cas particulier du $\mathrm{n}^{\circ} 10$ qui a régulièrement pris du poids tout au long de l'essai - $5,500 \mathrm{~kg}$ au total - cela représente un gain quotidien moyen de $91,7 \mathrm{~g}$, qui semble tout à fait remarquable.

L'évolution pondérale hebdomadaire du troupeau, traduite graphiquement a été la suivante :

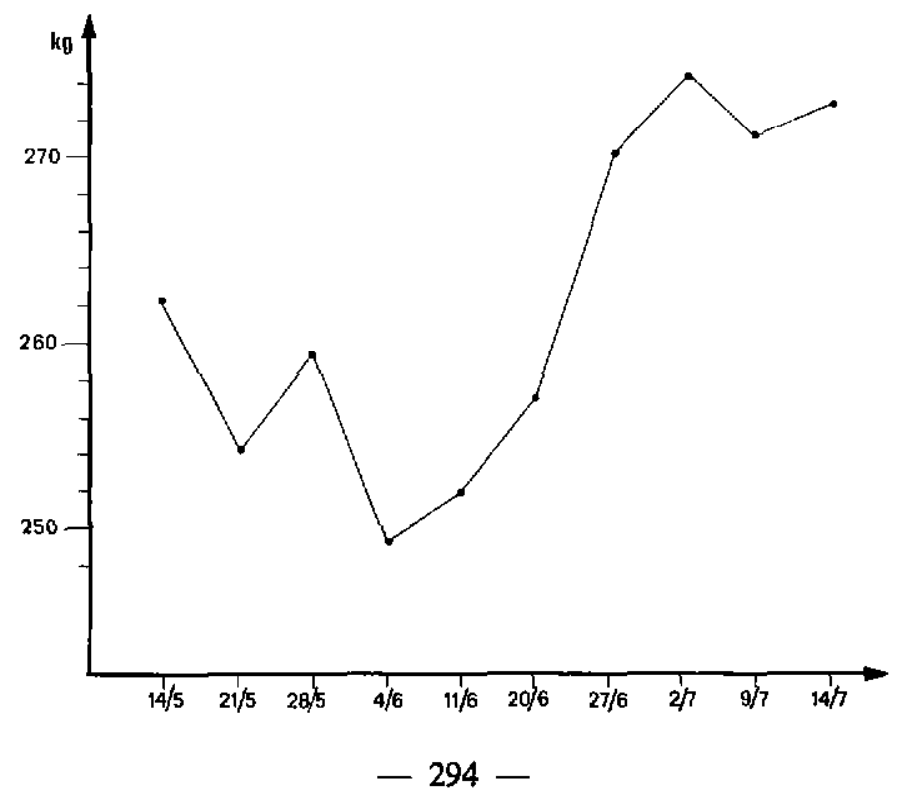


La ration offerte aux animaux ayant la composition suivante :
Ce tourteau constitue donc un important facteur de croissance et de développement lorsque

\begin{tabular}{|l|c|c|c|c|c|c|}
\hline \multirow{2}{*}{$\begin{array}{l}\text { Vigna sinensis } \\
\text { Niêbé }\end{array}$} & Poids (g) & M.S. (g) & M.A.D. (g) & U. F. & Ca (g) & P (g) \\
\cline { 2 - 7 } & 500 & 445 & 40,9 & 0,27 & 2,8 & 1,3 \\
\hline Foin d'Eragrostis & 500 & 470,5 & 0 & 0,20 & 1 & 0,18 \\
\hline $\begin{array}{l}\text { Tourteau } \\
\text { d'arachide }\end{array}$ & 100 & 93,3 & 41,2 & 0,1 & 0,1 & 0,5 \\
\hline Total & 1100 & 1008,8 & 82,1 & 0,57 & 3,9 & 1,98 \\
\hline
\end{tabular}

on voit que les besoins d'entretien ont été couverts, à peu de chose près avec les fanes de Niébé et le foin d'Eragrostis soit :
M. S. $\ldots \ldots \ldots \ldots \ldots \quad 915,5 \quad \mathrm{~g}$
U. F. . . . .
M. A. D. . . . $\ldots \ldots \quad 40,9$ g
Ca........... $3,8 \mathrm{~g}$
P $\ldots \ldots \ldots \ldots \ldots \ldots \quad 1,48 \mathrm{~g}$

Dans la ration donnée, la quantité de M. A. D. disponible a été le double de ce qui aurait été nécessaire, compte tenu de la richesse en M. A. D. de la fane de Niébé, qui est une légumineuse et du tourteau d'arachide.

Si ces fanes et le tourteau ont été consommés intégralement, il n'en a pas été de même pour le foin qui a fait l'objet d'un certain refus de la part des animaux, plus marqué en début qu'en fin d'essai.

Ce refus a été en moyenne de $500 \mathrm{~g}$ par jour, soit $50 \mathrm{~g}$ par animal ce qui fait qu'en définitive la ration moyenne ingérée par animal et par jour a été :
M. S......... $961,8 \quad \mathrm{~g}$
U. F. . . . . . $\ldots, 55$
M. A. D. . . . $\quad 82,1 \mathrm{~g}$
$\mathrm{Ca} \ldots \ldots \ldots \ldots \ldots \ldots \quad 3,8 \mathrm{~g}$
$\mathbf{P} \ldots \ldots \ldots \ldots \ldots \quad 1,96 \mathrm{~g}$

Les données de base de la ration n'ont donc pas été influencées par ce refus.

De ce qui précède, on peut conclure que le gain de poids global observé en fin d'essai au profit d'animaux dont l'état général était alors remarquable peut être attribué au tourteau d'arachide présent dans la ration. donné en supplément à une ration normale d'entretien, à raison de $100 \mathrm{~g}$ par jour et par animal.

Mais même à ce niveau, son prix d'achat - 22 F CFA le $\mathrm{kg}$ - le rend prohibitif pour le petit éleveur de chèvres rousses, alors que les fanes de Niébé, si elles ne sont pas produites par l'éleveur lui-même sont d'un prix très abordable lorsqu'elles sont achetées tôt dans la saison.

En conclusion, le tourteau d'arachide semble plus particulièrement indiqué pour assurer un complet et plus rapide développement des géniteurs sélectionnés destinés à l'amélioration des qualités naturelles de la chèvre rousse de Maradi.

\section{Rationnement avec des fanes de Niébé, du son} de mil et du tourteau d'arachide

\section{- Objet de l'essai}

Cet essai a eu pour but d'étudier les possibilités offertes par ces sous-produits pour assurer, en saison sèche, la croissance des jeunes caprins.

\section{- Conditions de l'essai}

Neuf jeunes boucs sevrés, âgés de 7 mois, d'un poids moyen de $14 \mathrm{~kg}$ en bon état ont été isolés pendant 2 mois, dans un parc de $600 \mathrm{~m}^{2}$ sans végétation avec de l'eau à volonté et une ration globale quotidienne de :

$$
\begin{array}{lll}
\text { Fanes sèches de Niébé } \ldots & 3,5 \mathrm{~kg} \\
\text { Son de mil } \ldots \ldots \ldots \ldots & 3,5 \mathrm{~kg} \\
\text { Tourteau d'arachide..... } & 1,7 \mathrm{~kg}
\end{array}
$$

Chaque animal a fait l'objet d'une triple pesée en début et en fin d'expérience, avec pesées hebdomadaires par groupe de 5 et 4 animaux du fait de leur indocilité individuelle. 


\section{— Résultats}

Les résultats globaux sont donnés ci-dessous :

Poids en $\mathrm{kg}$ :

- au 6 mai ........ 125,7

— au 6 juillet $\ldots \ldots \ldots \ldots \quad 162,2$

Gain de poids total.... $+36,5 \mathrm{~kg}$

et dans le graphique ci-après pour les variations hebdomadaires de poids.

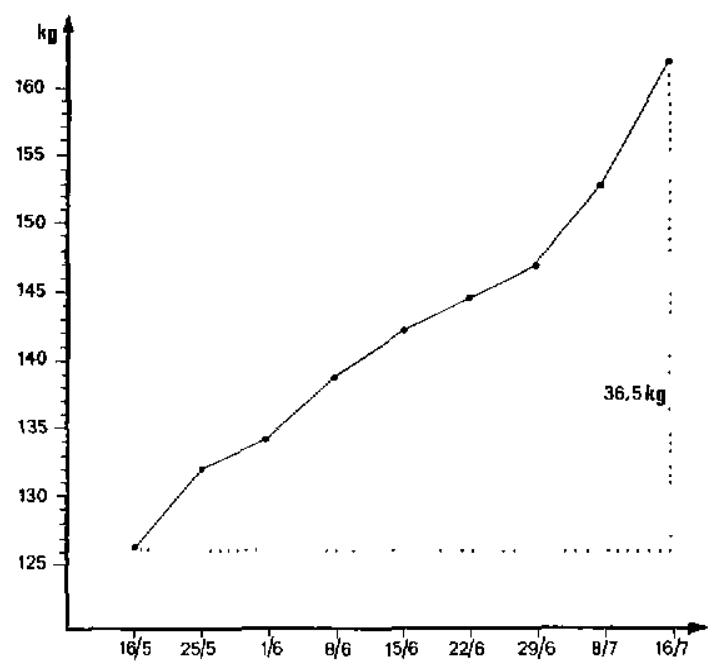

Ces données montrent que le poids des animaux a régulièrement progressé, le gain moyen par animal et par jour étant de 67,6 g pour les 2 mois d'observation, avec une brusque augmentation de $100,6 \mathrm{~g}$ au cours des seuls 15 derniers jours.

\section{- Discussion}

Pour les 9 boucs en observation, la composition de la ration offerte était, par animal et par jour, la suivante :
Pour assurer à un caprin de $15 \mathrm{~kg}$ un gain journalier de poids de $175 \mathrm{~g}$, les besoins théoriques sont :

Consommation $900 \mathrm{~g} \mathrm{M}$. S.

U. F. ....... 0,85 dont $\left\{\begin{array}{l}0,48 \text { U. F. pour } \\ \text { l'entretien } \\ 0,37 \text { U. F. pour } \\ \text { le gain de poids }\end{array}\right.$

$\begin{array}{lrr}\text { M. A. D. } \ldots . & 140 \mathrm{~g} \\ \text { Ca } \ldots \ldots \ldots & 2,8 \mathrm{~g} \\ \text { P } \ldots \ldots \ldots & 1,8 \mathrm{~g}\end{array}$

La ration offerte a donc couvert les besoins pour un gain théorique de poids de $100 \mathrm{~g} / \mathrm{jour}$. $\mathrm{Au}$ début, cette ration a été mal acceptée. Si les jeunes boucs ont parfaitement consommé les fanes de Niébé, ils ont refusé le son de mil et le tourteau d'arachide, pourtant broyé fin.

Ce n'est qu'au bout de 6 jours que le son de mil a été bien consommé mais il a fallu attendre 14 jours pour qu'il en soit de même avec le tourteau d'arachide.

Il convient de noter à ce sujet qu'à l'occasion de l'essai précédent, le même tourteau d'arachide a été parfaitement accepté par les boucs d'expérience, sans qu'il soit possible d'émettre la moindre hypothèse sur l'origine ou les raisons d'une telle différence de comportement.

Dans les conditions normales du Centre caprin de Maradi, les jeunes boucs âgés de 6 mois à 1 an, grossissent à la cadence moyenne de $35 \mathrm{~g}$ par animal et par jour.

Cet essai de rationnement est donc pratiquement satisfaisant puisqu'il a permis d'obtenir un gain de poids à peu près double, avec des animaux en état remarquable en fin d'observation.

Lorsque le son de mil est acheté dans le commerce ( $20 \mathrm{~F} \mathrm{CFA} \mathrm{le} \mathrm{kg)} \mathrm{le} \mathrm{coût} \mathrm{de} \mathrm{la} \mathrm{ration} \mathrm{indi-}$ viduelle journalière s'élève par animal à $16 \mathrm{~F}$ CFA, ce qui dépasse en général les possibilités financières des éleveurs.

\begin{tabular}{|l|c|c|c|c|c|c|}
\hline \multirow{2}{*}{ Fanes de Niếbé } & Poids (g) & M.S. (g) & N.A.D. (g) & U. F. & Ca (g) & P (g) \\
\cline { 2 - 7 } & 389 & 346,1 & 31,8 & 0,21 & 2,21 & 1 \\
\hline Son de mil & 389 & 359 & 32,3 & 0,31 & 0,3 & 1,72 \\
\hline $\begin{array}{l}\text { Tourteau } \\
\text { d'arachide }\end{array}$ & 189 & 176,3 & 78 & 0,19 & 0,2 & 0,9 \\
\hline Tota1 & 967 & 881,4 & 142,1 & 0,71 & 2,71 & 3,62 \\
\hline
\end{tabular}

Par contre, lorsque ce son est un des sousproduits des activités culturales des propriétaires de chèvres, comme c'est le cas le plus souvent, le prix de cette ration s'abaisse aux environs de 7,5-8 F CFA le kg qui est économiquement rentable. 


\section{CONCLUSION}

Les essais de rationnements faits en saison sèche avec des sous-produits de l'agriculture locale (graines de coton, fanes sèches de Niébé, son de mil et tourteau d'arachide) pour permettre aux caprins de la race rousse de Maradi de supporter au mieux la disette pastorale de fin de saison sèche ont montré :

- que la graine de coton entière, non délintée est capable d'assurer, à elle seule, et à des conditions économiques très acceptables l'entretien de ces animaux ;

- que la fane de Niébé et du foin d'Eragrostis constituent une bonne ration d'entretien. Additionnée de tourteau d'arachide à raison de $100 \mathrm{~g}$ par jour et par animal, elle se transforme en ration de développement de qualité pour des boucs adultes n'ayant pas encore atteint leur poids maximal ;
- que des fanes de Niébé, du son de mil et du tourteau d'arachide constituent ensemble une ration de croissance remarquable pour des jeunes boucs entre 6 mois et 1 an.

Le prix de revient de ces 2 dernières rations est toutefois d'un prix prohibitif pout les agriculteurs-éleveurs qui ne produisent pas eux-mêmes les fanes de Niébé et le son de mil qui leur sont nécessaires, d'autant plus que le tourteau d'arachide, de production industrielle, est lui-même d'un prix au $\mathrm{kg}$ relativement élevé - ce qui semble en réserver l'utilisation à l'alimentation des reproducteurs sélectionnés.

Ces essais ont été d'autant plus concluants que les boucs étaient en fin d'expérience dans un état général supérieur à celui des boucs du troupeau du Centre qui, disposant de pâturages assez pauvres de saison sèche, étaient supplémentés avec des fanes sèches de Niébé à raison de $800 \mathrm{~g}$ par animal et par jour.

\section{SUMMARY}

\section{Rationing experiment on Red Sokoto goats using local agricultural by-products}

Local agricultural by-products can be used in the feeding of the Red Sokoto goats in Maradi (Niger) in order to bridge the gap at the end of the dry season.

a) Whole cotton seeds with their linters are sufficient in themselves to provide goats with feeds without any digestive troubles and at a competitive rate for breeders.

b) Dry Niébé jaulms mixed with Eragrostis hay constitute a good and cheap maintenance feed ration which can be tumed into a good growth feed ration by adding groundnut cake. A mixture of Niébé haulms, millet bran and groundnut cake is a very good ration for growth in young animals.

Groundnut cakes only are too expensive to be currently mixed into the feeding ration of the Red Sokoto goat.

\section{RESUMEN}

Ensayos de racionamiento de machos cabrios de la raza rojiza de Maradi (Niger) con subproductos de la agricultura del país

En Maradi (Niger), los subproductos de la agricultura del país pueden asegurar la alımentación de la cabra rojiza al fin de la estación seca.

a) la semilla de algodón, entera, con su borra, basta sola para mantener los animales. sin causar trastornos digestivos y con condiciones aceptables por los ganaderos.

b) Las hojarascas de Niebe y el heno de Eragrostis constituyen juntos una ración de mantenimiento buena y económica que se transforma en buena ración de crecimiento por adición de torta de cacahuete.

Las hojarascas de Niebé, el salvado de mijo y la torta de cacahuete mezclados representan una muy buena ración de crecimiento para las crías.

Sola la torta de cacahuete es demasiado cara para utilisarse corrientemente en la alimentación de la cabra de Maradi.

\section{BIBLIOGRAPHIE}

1. RIVIERE (R.). Manuel d'alimentation des ruminants domestiques en milieu tropical. $1^{\text {re }}$ éd., Paris, Ministère Coopération ; Maisons-Alfort, 1. E. M. V. T. 1977.
2. DEVENDRA (C.), BURNS (M.). Goat production in the tropics. Farnham Royal (G. B.), Commonwealth Agricultural Bureaux, 1970. 
3. QUITTET (E.). La chèvre - Le guide de l'éleveur. Paris, Maison Rustique, 1975.

4. ROBINET (A. H.). La chèvre rousse de Maradi. Son exploitation et sa place dans l'économie et l'élevage de la République du Niger. Rev. Elev. Méd. vét. Pays trop., 1967, 20 (1) : 129-186.

5. ROBINET (A. H.). La chèvre de Maradi et l'élevage caprin au Niger. Paris, S. E. A. E., 1971.

6. RÓBINET (A. H.). La chèvre de Maradi et l'élevage caprin au Niger. Courrier Association, 1972 (12): 32-37.
7. HAUMESSER (B.). Quelques aspects de la reproduction chez la chèvre rousse de Maradi. Comparaison avec d'autres races tropicales ou subtropicales. Rev. Elev. Méd. vét. Pays trop., 1975, 28 (2) : 225-234.

8. MACKENZIE (D.). Goat husbandry. London, Faber and Faber, 1967.

9. POUDELET (E.). Contribution à l'étude de la chèvre rousse de Maradi. Paris, Copedith, 1976. Thèse. Méd. vét., Alfort. 1976, n 101. 